\title{
Machine learning: technologies and potential application at mining companies
}

\author{
Snizhana Zelinska \\ Kryvyi Rih State Pedagogical University, 54 Gagarin Ave., Kryvyi Rih, 50086, Ukraine
}

\begin{abstract}
Implementation of machine learning systems is currently one of the most sought-after spheres of human activities at the interface of information technologies, mathematical analysis and statistics. Machine learning technologies are penetrating into our life through applied software created with the help of artificial intelligence algorithms. It is obvious that machine learning technologies will be developing fast and becoming part of the human information space both in our everyday life and in professional activities. However, building of machine learning systems requires great labour contribution of specialists in the sphere of artificial intelligence and the subject area where this technology is to be applied. The article considers technologies and potential application of machine learning at mining companies. The article describes basic methods of machine learning: unsupervised learning, action learning, semi-supervised machine learning. The criteria are singled out to assess machine learning: operation speed; assessment time; implemented model accuracy; ease of integration; flexible deployment within the subject area; ease of practical application; result visualization. The article describes practical application of machine learning technologies and considers the dispatch system at a mining enterprise (as exemplified by the dispatch system of the mining and transportation complex "Quarry" used to increase efficiency of operating management of enterprise performance; to increase reliability and agility of mining and transportation complex performance records and monitoring. There is also a list of equipment performance data that can be stored in the database and used as a basis for processing by machine learning algorithms and obtaining new knowledge. Application of machine learning technologies in the mining industry is a promising and necessary condition for increasing mining efficiency and ensuring environmental security. Selection of the optimal process flow sheet of mining operations, selection of the optimal complex of stripping and mining equipment, optimal planning of mining operations and mining equipment performance control are some of the tasks where machine learning technologies can be used. However, despite prospectivity of machine learning technologies, this trend still remains understudied and requires further research.
\end{abstract}

Topicality of the research consists in the fact that at present many mining companies are facing the necessity of increasing efficiency of their performance in conditions of rising competitiveness and market globalization. To achieve the set goals, it is essential to reduce costs, optimize use of equipment and human resources.

Mining companies often implement technological process control systems where a great amount of current information on their production processes is stored. This information is used directly for operating control and management. The practical value of the accumulated information in the long term mainly consists in statistical reporting.

In view of the above, a need arises to use the accumulated information for identifying segments of technological processes of less efficiency. To do this, means of machine learning technologies may be used.

Machine learning focuses on development of applications and specialized algorithms capable of selflearning and adapting. Application of machine learning algorithms enables processing data to search for relevant patterns and change programme actions.

The article aims to study technologies and potential of machine learning at mining companies.

To reach the set research objective, the following tasks should be accomplished:

- to reveal the essence of machine learning;

- to describe machine learning algorithms;

- to provide an example of a subject area of machine learning application at mining companies.

Despite the tremendous development of machine learning technologies in the recent decade, artificial intelligence remains a rather ambiguous concept. It includes a number of subject areas: from generation of plausible images on a certain theme to prediction of time series. Methods of machine learning are the basis of artificial intelligence technologies but remain a highly tailored solution to every particular task.

Machine learning is a sub-division of artificial intelligence dealing with methods of elaborating algorithms capable of finding dependencies in data and

\footnotetext{
* Corresponding author: zvit-zss@ukr.net
} 
learning. Learning from examples is based on identification of patterns by individual empirical data. Deductive learning is implemented through formalization of expert knowledge and its transfer to the knowledge database [3].

S. A. Shumskiy suggests creating strong artificial intelligence using principles of the human brain work and does a review of the current state and prospects for further investigations into machine intelligence [12]. Only machine learning supported by the achieved level of computer capacities is able to make machine intelligence fast soar to the human level and engage these capacities into solving the problems only people can manage.

Methods of machine learning include: supervised learning, unsupervised learning, action learning, semisupervised machine learning [11].

Supervised learning is implemented through inputting data into a machine and their preferable outputting, objects called "a teacher". Its objective is to study the general rule which will enable displaying input data at output. Algorithms like that use everything they have learned earlier when dealing with any new data.

Many types of learning are based on the generalizing transition from training data to new data through studying similarities between them. In grouping models, which include, in particular, decision trees, such similarity takes on the form of equivalence relation, or object-space splitting: two objects are considered similar if they are in the same segment of splitting [10].

Unsupervised learning assumes that labels, tags or explanations are not transferred to the learning algorithm with respect to the input, and it remains as it is, for searching for a relevant structure in it. Such algorithms are used to find hidden patterns in data arrays and draw one's own conclusions or conclusions from the data obtained from data sets.

In practice, learning is implemented through direct interaction with the changing environment and is to fulfil a certain task (e.g. operating a vehicle, equipment positioning) without informing whether it is reaching the destination or not.

Semi-supervised leaning means that the subject "teacher" gives the machine data with certain drawbacks, and results are not obtained.

Use of a particular method of machine learning is conditioned by the subject area of its practical application. The most preferable methods of machine learning are supervised learning, unsupervised learning that enable solution of most tasks of mass data processing.

Matrices, graphs, probability, function, gradient, minimum of function, optimization, error are the most popular objects of neuromathematics that are frequently used in machine learning.

The array of source data in a particular neural network is the most illustrative example of practical application of a matrix (a table of numbers) [5]. Fig. 1 presents the generalized chart of a single-layer perceptron that recognizes digits.

The perceptron consists of three types of elements, namely: incoming signals from sensors, associative elements and responders. Thus, perceptrons allow creating a set of "associations" between incoming stimuli and the required output response.

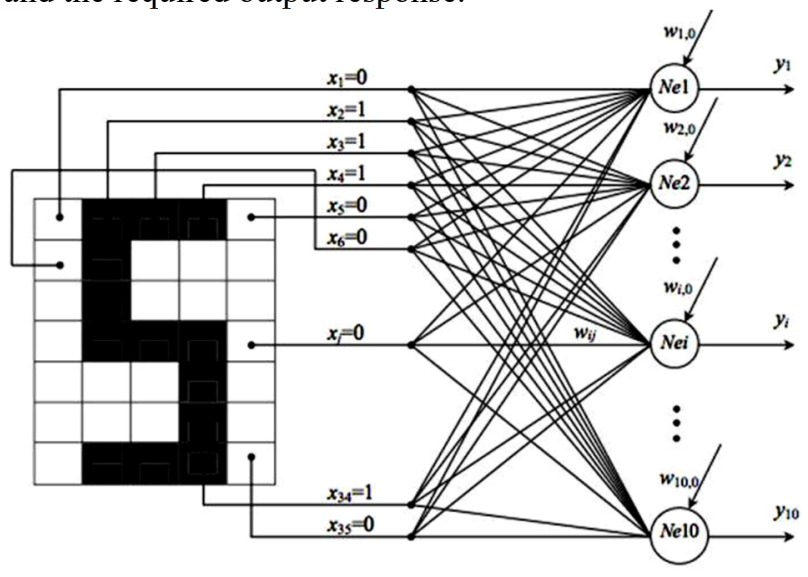

Fig. 1. A single-layer perceptron.

In the modern terminology, perceptrons may be classified as artificial neural networks:

- with a single hidden layer;

- with a threshold transfer function;

- with direct signaling.

Another object, a graph, is a set of vertices and a corresponding set of connections between them. It is a good visual mathematical object depicting a model chart or an algorithm structure.

In mathematics, a graph is often represented by an ordered pair $G=(V, E)$, where $V$ is a set of vertices, $E$ is a set of edges linking these vertices.

The given diagram shows that the source image may be presented as a corresponding 7-by-5 matrix consisting of ones and zeros, Fig. 2.

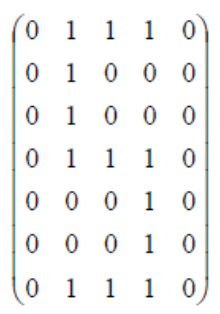

Fig. 2. A matrix of perceptron values.

Various calculations that can be performed on such a graph enable finding, for instance, the shortest bypass road or the nearest handling facilities, planning the optimal haulage route, etc.

Description of links between objects with the help of graphs is implemented through associating edges to certain quantitative values, qualitative attributes or required characteristics called weights. In the simplest case, this can be an edge sequence numbering showing the order of their direct consideration (priority or hierarchy of events).

The graph edge weight may depict:

- the haulage route length (transportation);

- bandwidth (communication);

- voltage or current strength (electric circuits);

- the conductor colour in the wiring diagram of an electronic device; 
- the amount of work completed (production);

- link valences (chemical formulas), etc.

When assessing machine learning, the following requirements are considered: speed of operations; assessment time; implemented model accuracy; ease of integration; flexible deployment within the subject area; ease of use; result visualization.

Application of machine learning technologies in the mining industry is a promising and necessary condition for increasing mining efficiency and ensuring environmental security. However, despite prospectivity of machine learning technologies, this trend still remains understudied and is not yet implemented to the required extent.

According to M. B. Nosyrev, A. V. Druzhinin and N. B. Glushenko, increased efficiency of mining companies' performance depends on arrangement of coal mining by the open pit method. Unique machinery used here and sophisticated technologies require practical application of intelligent technologies and simulation of mathematical models when designing, planning and controlling the life cycle of a coal pit [6].

One of the solutions to these tasks consists in transferring the expert system technology into the artificial intelligence sphere of industrial information management systems. Traditional problem areas for expert systems are: identification and diagnostics used for situation interpretations; prediction used for determining probable sequences of the identified situations and states; planning for elaborating goals and adaptive control for achieving the set goals.

Here, application of machine learning technologies enables obtaining qualitatively new knowledge based on which the following tasks can be accomplished:

- selection of the optimal process flow sheet of mining operations;

- selection of the optimal (by basic characteristics) complex of stripping and mining equipment;

- optimal operational planning of mining operations and mining equipment performance control;

- useful minerals quality;

- life of mine;

- completeness and quality of useful mineral extraction and processing;

- mine field sizes;

- the designed depth of open pit and underground operations;

- pmining elements sizes (level height, sizes of blocks, panels, rooms);

- the period of construction and exploitation of the designed deposit thickness.

The above classification shows that current formal prediction and control models in mining cannot always be adequately applicable at a mining enterprise and cause the necessity of developing new approaches to creating systems on the basis of machine learning technologies.

For instance, V. N. Soloviev et al. investigates methods and algorithms used at solving problems of assessment, regression and filtration, image recognition and clusterization [8]. The considered classic and modern implementations of machine learning algorithms can be applied to solving tasks of processing data of a particular subject area.

A. O. Zibert and V. V. Miroshnichenko set the task of applying machine learning algorithms to increase efficiency of truck performance. They provide description and give an example of practical application of the perceptron model to predicting performance periods without maintenance-caused outages based on the classification of such performance periods [4].

The authors also provide detailed description of training data and the method of generating these data from the database, consider the results of the perceptron model application and conclude on possible application of the used model to solve machine learning tasks.

The intellectual mining-engineering system should continuously identify and monitor characteristics of a controlled object. Feedback and availability of a reliable automated control system are also required. Such systems are always controlled under conditions of principal uncertainty of development and incomplete observability. Building formal models for most processes of a mining company is extremely complicated.

In view of the above, it is necessary to implement machine learning systems at mining companies considering interaction of various production components including engineering, technological and socioengineering mining objects.

According to I. O. Temkin and A. N. Goncharenko, there currently exist many intellectual models, methods and technologies widely used in controlling complex industrial objects and socio-engineering systems: data mining; fuzzy logic; probabilistic prediction models; neural network models of situation recognition; genetic algorithms; knowledge acquisition; natural language processing; search for solutions in conditions of insufficient information provision [9].

Another problem that can be solved with the help of machine learning is engineering research during building of complex engineering systems (F. N. Abu-Abed [1, 2]). Timely recognition of pre-emergency situations that can occur during operations may provide production stability and safety. A pre-emergency situation is an emerging process different from the regular one and requiring a prompt response. The largest problems in timely detection and identification of pre-emergency situations occur in a complex engineering system during its building.

Examples of complex engineering plants include drilling rigs for oil and gas wells drilling, radars and other complex engineering systems. Complications are caused by:

- the necessity of operational visual control over current technological processes in the system;

- the necessity of making relevant decisions and subsequent production of recommendations in the realtime mode in conditions of insufficient information;

- the necessity of operational accounting of a large quantity of initial data and diverse factors;

- insufficient qualification of some enterprise employees $[1 ; 2]$.

In recognizing pre-emergency situations occurring during the trial operation of systems, the number of attribute space dimensions is rather big and boundaries 
between pre-emergency situation classes are blurred. Application of cluster analysis and Bayesian classification to solving the task of pre-emergency situations recognition during well drilling is not reasonable. With this purpose, artificial feedforward neural networks capable of being taught through practical use of the back propagation algorithm may be used as a mathematical tool for solving the pre-emergency situations recognition task.

Many mining companies apply dispatch systems and machinery monitoring. The automatic system of controlling mining and transportation complex "Quarry" of VIST Group is an example of such a system [7]. Fig. 3 presents the system architecture.

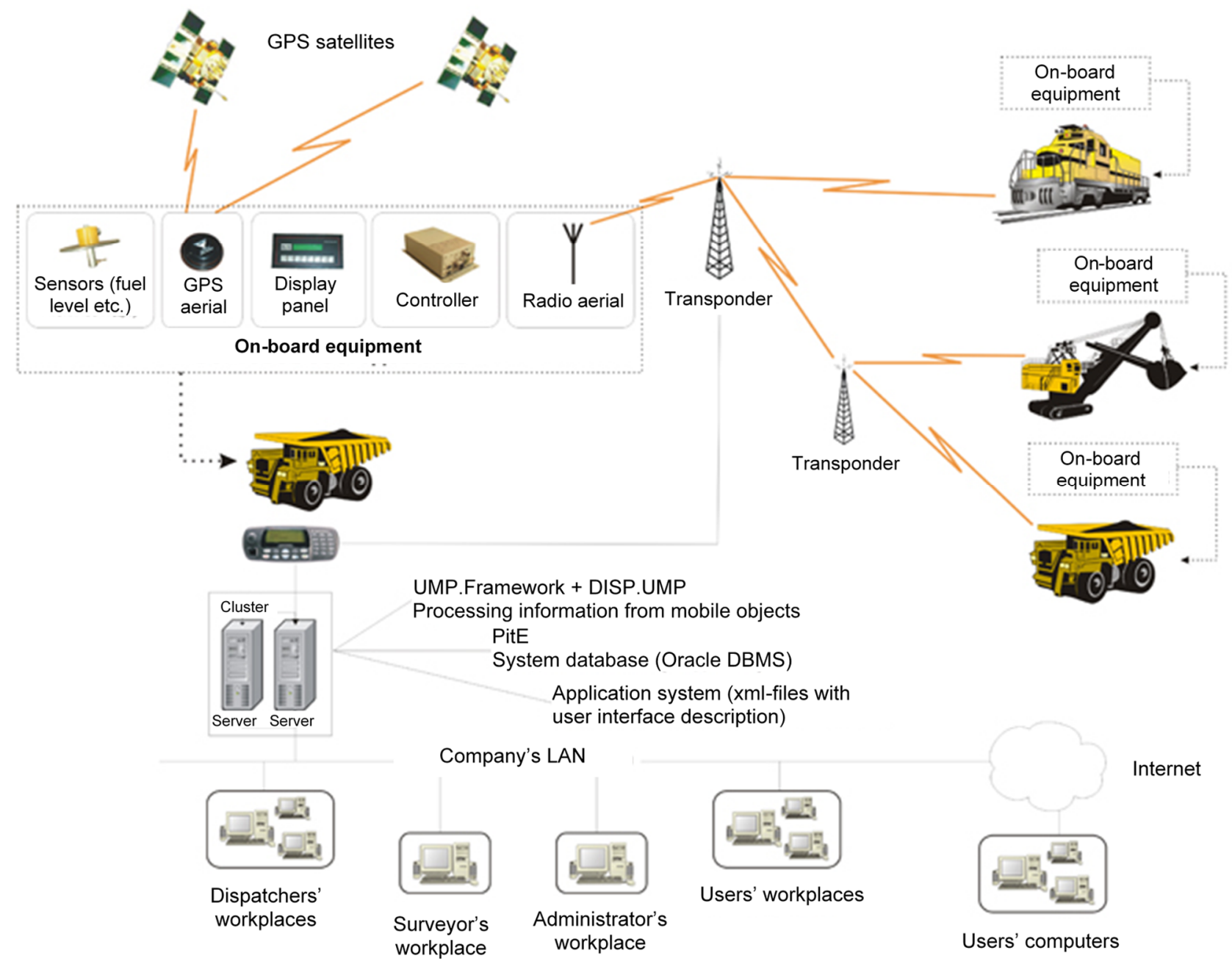

Fig. 3. Architecture of the automatic system "Quarry".

The automatic system "Quarry" receives data from the following sensors:

- sensors of machinery position, speed and route (GPS);

- handled ore mass weight sensors;

- excavator bucket position sensors;

- pressure sensors in the bulldozer turbine;

- truck wheel pressure sensors;

- excavator operation currents sensors;

- fuel level sensors.

The information users are:

- the dispatcher control room of an enterprise, various users of the enterprise local network;

- other engineering services of an enterprise statistics on real equipment performance, stand-by time, kilometers travelled, handled material volumes.

Besides, it is possible to detect equipment stand-by time or determine periods of its most efficient performance on the basis of the collected information.

However, due to a great amount of the information processed, analysis of historical data is rather labour consuming. To eliminate the problem, various data processing algorithms should be used to enable prompt analysis of causes of inefficient equipment use and search for ways of reducing the number of and preventing such situations.

As the initial data for the set goal of increasing efficiency of mining equipment use are rather similar to the presented examples of machine learning use, one may say about use of machine learning algorithms for determining the optimal mode of mining equipment performance based on dispatch system data as well as determining factors impacting efficiency of mining equipment use.

To estimate the above assumption, one can use the perceptron-based algorithms of machine learning through 
comparing the obtained predictions with the real data. The perceptron consists of three types of elements: signals from sensors, associative elements and responders. Thus, perceptrons enable creation of a set of "associations" between the incoming stimuli and the required output response.

The perceptron performance may aim at creating the following classification of mining equipment:

- class 1 - probability of mining equipment stand-by caused by possible maintenance within the following 30 days makes $100 \%$;

- class 2 - probability of mining equipment stand-by caused by possible maintenance within the following 60 days makes $100 \%$;

- class 3 - probability of mining equipment stand-by caused by possible maintenance within the following 90 days makes $100 \%$;

- class 4 - probability of mining equipment stand-by caused by possible maintenance within the following 120 days makes $100 \%$;

- class 5 - probability of mining equipment stand-by caused by possible maintenance within the following 150 days makes $100 \%$;

- class 6 - probability of mining equipment stand-by caused by possible maintenance within the following 180 days makes $100 \%$.

The following information on mining equipment stored in the dispatch system database may be input into the perceptron model:

- average rate of mining equipment performance $(\mathrm{km} / \mathrm{h})$ per shift;

- average energy consumption per shift; shift;

- average inclinometer reading value (degrees) per

- average temperature (degrees centigrade) per shift.

However, mining production possesses a number of specific features that condition complexity of implementation of formal methods and models of control.

The first and the most significant feature of a mining company is production which is discrete-continuous in character. The second but not less important feature is a specific controlled object - nature (parameter distributability, process stochasticity, etc.). Another feature is specificity of the control system which includes complex functional blocks, such as survey, geological, etc.

Thus, based on the obtained results, the following conclusion can be drawn: it is possible to apply machine learning algorithms to enhancing efficiency (in the medium term at least).

Besides, further investigations are required, in particular into application of other machine learning algorithms and enhancement of initial data for obtaining better results of an algorithm operation. Another direction of research is application of machine learning technologies to prediction of not only stand-by periods but also efficiency of a mining equipment unit in the short, medium and long term, etc.

\section{References}

1. F. N. Abu-Afed, Dissertation, Tver State Technical University, 2011

2. F. N. Abu-Afed, Territoriia neftegaz. Burenie 6, 1619 (2012)

3. O. Markova, S. Semerikov, M. Popel, CEUR Workshop Proceedings 2104, 388-403 (2018), http://ceur-ws.org/Vol-2104/paper_204.pdf. Accessed 30 Mar 2020

4. A.O. Zibert, V.V. Miroshnichenko, Universum: Tekhnicheskie nauki 2(24) (2016), http://7universum.com/ru/tech/archive/item/2968. Accessed 15 Dec 2019

5. S.O. Semerikov, I.O. Teplytskyi, Yu.V. Yechkalo, A.E. Kiv, CEUR Workshop Proceedings 2257, 122 147 (2018), http://ceur-ws.org/Vol2257/paper14.pdf. Accessed 21 Mar 2020

6. M.B. Nosyrev, A.V. Druzhinin, N.V. Glushenko, Izvestiia Uralskogo gosudarstvennogo gornogo universiteta 7, 165-168 (1998)

7. Zifra Mining, Open-pit mining (2020), https://vistgroup.ru/solutions/open-pit-mining/asuscc-quarry/. Accessed 21 Mar 2020

8. A.O. Tarasenko, Y.V. Yakimov, V.N. Soloviev, CEUR Workshop Proceedings 2546, 101-114 (2019)

9. I. O. Temkin, A. N. Gonchrenko, Nauchnotekhnicheskie vedomosti Sankt-Peterburgskogo gosudarstvennogo politekhnicheskogo universiteta 42 (183), 252-258 (2013)

10. P. Flach, Machine Learning: The Art and Science of Algorithms that Make Sense of Data (Cambridge University Press, Cambridge, 2012). doi:10.1017/CBO9780511973000

11. S.O. Semerikov, I.O. Teplytskyi, Metodyka uvedennia osnov Machine learning u shkilnomu kursi informatyky (Methods of introducing the basics of Machine learning in the school course of informatics), in Problems of informatization of the educational process in institutions of general secondary and higher education, Ukrainian scientific and practical conference, Kyiv, October 09, 2018 (Vyd-vo NPU imeni M. P. Drahomanova, Kyiv, 2018), pp. 18-20

12. S.A. Shumskii, Mashinnyi intellekt. Ocherki po teorii mashinnogo obucheniia $i$ iskusstvennogo intellekta (Machine intelligence. Essays on Theory of Machine Learning and Artificial Intelligence). (RIOR, Moscow, 2019) 